\title{
Physical Activity and the Prevalence of General and Abdominal Obesity among Saudi Women of Reproductive Age in Jeddah City
}

\author{
Alharbi $\mathrm{MM}^{\star}$ and Robert T Jackson \\ Department of Nutrition and Food Science, Skinner Hall, University of Maryland, College Park, MD, USA
}

*Corresponding author: Alharbi MM, Department of Nutrition and Food Science, 0119C Skinner Hall, University of Maryland, College Park, MD USA 20742-7521, Tel: 240-463-5044, E-mail: malharbi.sa@gmail. com

Citation: Alharbi MM, Robert T Jackson (2017) Physical Activity and the Prevalence of General and Abdominal Obesity among Saudi Women of Reproductive Age in Jeddah City. J Obes Overweig 3(1): 104. doi: 10.15744/24557633.3.104

Received Date: March 10, 2017 Accepted Date: May 16, 2017 Published Date: May 18, 2017

\begin{abstract}
Background: Obesity and physical inactivity are growing problems that are associated with major health problems. However, the current information on the association between obesity and physical activity (PA) in Saudi women of reproductive age is insufficient to design and develop intervention programs.

Objective: To explore the rates of two types of obesity (general and abdominal obesity) and the level of PA and to evaluate the relationship among obesity risk factors, the use of exercise equipment at home, and obesity measurements.

Design: A cross-sectional study was conducted in 2014 using a stratified two-stage cluster sample. The sampling weight and design effect were incorporated into the analysis. Body mass index (BMI) and waist circumference (WC) data were collected from all participants.

Setting: Twelve primary health care centers (PHCCs) in Jeddah, Saudi Arabia.

Subjects: A total of 408 Saudi women between 15 and 49 years of age.

Results: Of the 408 women evaluated, $33.8 \%$ were obese (BMI $\geq 30 \mathrm{~kg} / \mathrm{m}^{2}$ ), $25.1 \%$ were abdominally obese (WC $\geq 88 \mathrm{~cm}$ ), $47.1 \%$ were abdominally obese (WC $\geq 80 \mathrm{~cm}$ ), and $31.2 \%$ were physically inactive. There were significant associations between obesity measurements and family history of chronic diseases. No significant associations were found between PA levels and either type of obesity and the mean WC. As might be expected, women who had and used the exercise equipment were significantly more physically active than those who had it but did not use it; however, no significant differences were found between those women based on BMI and WC levels.

Conclusion: The prevalence of general obesity, abdominal obesity, and physical inactivity were remarkably high in Saudi women of reproductive age attending Jeddah PHCCs. Therefore, an intervention program to combat obesity is greatly needed, especially one that focuses on PA and mitigates social norms.

Keywords: Obesity; Abdominal Obesity; Physical Activity; Saudi Women; Reproductive-Aged Women

List of Abbreviations: PA: Physical Activity; BMI: Body mass index; WC: Waist Circumference; PHCCs: Primary Health Care Centers; NCDs: Noncommunicable Diseases; PSU's: Primary Sampling Units; PPS: Probabilities Proportional to their Size; IPAQ: International Physical Activity Questionnaire; MET-Min/Week: Metabolic Equivalent Task Minutes per Week; WHO: World Health Organization standards; IDF: International Diabetes Federation
\end{abstract}

\section{Introduction}

Obesity is one of the ten leading health indicators used to measure health status [1]. Worldwide, the prevalence of obesity is generally higher among women than among men; nearly two thirds of reproductive-aged women in the United States are currently overweight or obese [2], placing them at elevated risk for adverse health outcomes. A recent Saudi National Health Survey in people aged 15 years and above indicated that the prevalence of obesity was significantly higher among women (33.5\%) than men (24.1\%) [3]. This survey also showed high rates of noncommunicable diseases (NCDs) among Saudi women, such as diabetes, hypertension, and hypercholesterolemia. The survey found that $11.7 \%$ of the women had diabetes, $12.5 \%$ had hypertension, and $7.3 \%$ had hypercholesterolemia (40\% of the study samples were between the ages of 15 and 24) [4].

The reproductive years are an important life stage for women; it may result in significant weight gain that could affect women's short- and long-term health and the health of their children [5,6]. However, as most studies in Saudi Arabia have focused on the male population, children, adolescents, and women in their college years (under the age of 24 years), existing literature in Saudi Arabia focusing on the prevalence of obesity among Saudi women of reproductive age is lacking. Additionally, most of the existing 
studies in Saudi Arabia have used BMI to measure the obesity levels, failing to incorporate WC measurements, which has been shown to be a strong predictor of total body fat, adipose tissue [7-9], and obesity-related health risk [10].

Several studies have shown that performing physical activity (PA) on a regular basis (at least 30 minutes of moderate PA per day) enhances quality of life and health conditions [11]. In Saudi Arabia, low levels of PA and a sedentary lifestyle have become the norm among the Saudi population [12]. According to data from the Saudi National Health Survey (2013) [3], the prevalence of physical inactivity was high among the Saudi population (15 years of age and older), revealing significantly higher levels of inactivity in women (75.1\%) than men (47.0\%). However, information on the levels of PA and other lifestyle practices in Saudi adult women is lacking, especially among women particularly at risk of weight gain, such as those of reproductive age.

Furthermore, most of the current studies in Saudi Arabia and in Middle East countries have used the World Health Organization (WHO) standards for determining general $(\mathrm{BMI} \geq 30)$ and abdominal $(\mathrm{WC}>88 \mathrm{~cm})$ obesity. Therefore, in order to compare and interpret our results in relation to the previous studies, we decided to use WHO criteria to measure general and abdominal obesity. Also, to compare our results with worldwide literature, we determined the abdominal obesity using another WC cut-off point $(\mathrm{WC}>80 \mathrm{~cm}$ ), which is the International Diabetes Federation (IDF) criteria. Since, the new harmonized guidelines for the diagnosis of metabolic syndrome had recommended using the European cut-off points (IDF) for the Eastern Mediterranean Region until more specific data are available [13].

As far as it is known, no study has explored the prevalence of obesity and PA among Saudi women of reproductive age in Jeddahthe most liberal, urban, and diverse city in Saudi Arabia where the prevalence of obesity is high [14]. To fill the gap in the literature, this study was conducted in Jeddah, using a representative sample of Saudi women (15-49 years) who attended services at Jeddah primary health care centers (PHCCs). The aim was to explore the rates of two types of obesity (general and abdominal obesity) and the levels of PA and to evaluate the relationship among obesity risk factors, the use of exercise equipment at home, and obesity measurements. This information may help design strategies and interventions to help prevent and control obesity and to increase PA among Saudi women who received services at Jeddah PHCCs.

\section{Methods}

\section{Sample}

The study was conducted using a cross-sectional stratified two-stage cluster sampling design survey of 408 Saudi women, aged 1549 years, who attended general clinics at JPHCCs. The sampling procedure aimed to select a representative sample of women who were only seeking services at PHCCs in Jeddah City. Clinics served as Primary Sampling Units (PSU's) and were grouped into four health sector strata. In the first stage, the PHCCs (clusters) were sampled without replacement and selected (12 out of 37 centers) with Probabilities Proportional to their Size (PPS), from the list of PHCCs in survey area. The second sampling stage involved recruitment of women from the selected PHCCs (12 centers) using a systematic sampling procedure from the eligible women attending on days the sampling PHCCs (General Clinics) were visited. The first woman participant who fulfilled the inclusion criteria was invited to enroll in the study. Then, every third attending woman who fulfilled the criteria was selected and soon until completion of the required sample from the PHCC was achieved. However, if a selected woman did not fulfill the inclusion criteria (exclusion criteria) or refused to participate in this study, then selection proceeded to the next sample woman attending the PHCC. Subjects who were pregnant/ lactating and those having serious diseases (e.g., organ failure, transplant, ascites, cancer, and mental illness), were excluded.

Sample size was determined by performing an a-priori power analysis to determine the number of participants required to detect a small effect of design $\left(f^{2}=0.1\right)$ with power $=.80$, and at $\alpha=.05$ (the power analysis was conducted with $G^{\star}$ Power 3.1.4.). The analysis indicated a sample size of 201 would be sufficient. Then, this was adjusted for clustering by multiplying this sample size by a convenient design effect of 2.0 , which indicated that a minimum of 402 women would be sufficient to accurately estimate results for the final sample. However, to select an equal number of the women from the selected health centers (12 centers), we increased the sample size to 408 ( 402 women $/ 12$ centers $=33.5$ women $\approx 34$ per center).

\section{Survey}

Participants were asked to participate in answering the survey questions as volunteers. The informed consent information was distributed to participants before participating in the study. A structured questionnaire (face-to-face interview) was used in the survey to elicit information on the socio-demographic characteristics, medical and history of chronic diseases, obstetric history, $\mathrm{PA}$, and lifestyle information.

\section{Physical Activity Assessment}

PA was assessed using the official Arabic short version of the International Physical Activity Questionnaire (IPAQ) [15]. IPAQ was subjected to a reliability and validity study carried out in 14 centers in 12 countries during the year 2000, and demonstrated reasonable test-retest reliability (intra-class correlations range $0.7-0.8$ and inter-method validity (median $r_{s}=.67$ ) [16]. The short form of IPAQ has been validated and used by numerous studies among the Saudi Arabia adult population $[3,12,17-19]$. The total 
weekly PA (Metabolic Equivalent Task minutes per week (MET-Min/week)) was calculated by multiplying the number of minutes spent in each activity category (low, moderate, and vigorous levels) with the specific MET score for each activity. The MET intensity values that were used to score IPAQ questions were: vigorous (8 METs), moderate (4 METs) and low (3.3 METs) [20]. For descriptive analysis, the PA scoring was categorized as: physically inactivate (low activity <600 MET-min. /week), and physically active (sufficient activity $\geq 600$ MET-min. /week).

\section{Anthropometric Measurements}

Weight and height were measured on a digital scale with stadiometer (Seca 703 medical scale) (Hamburg, Germany). Weight was recorded to the nearest $100 \mathrm{gm}$ and height to the nearest $0.1 \mathrm{~cm}$. Measurements of participants were collected in a private area (exam room) and instructed them to remove excess clothing, overcoats, Hejab, Abaya, and shoes.

BMI was calculated as weight divided by height squared $\left(\mathrm{kg} / \mathrm{m}^{2}\right)$, and was stratified for the purpose of analysis into two categories: non-obese and obese based on World Health Organization standards (WHO) 2012 [21]. For adult ( $\geq 20$ years old), non-obese group was defined as $\mathrm{BMI}<25 \mathrm{kgm}^{2}$, and obese group as $\mathrm{MBI} \geq 25 \mathrm{kgm}^{2}$. For adolescents ( $\leq 19$ years old), non obese group was defined as BMI $<85$ th age-specific percentile, and obese group as BMI $\geq 85$ th age-specific percentile. Online software was used to calculate BMI age-specific percentile from height, weight, age, and sex data, based on WHO reference populations [22,23].

WC was measured at the mid-point between the iliac crest and lowest rib to the nearest $0.1 \mathrm{~cm}$. Then, abdominal obesity was defined using two cut-offs; WC $>88 \mathrm{~cm}$ according to WHO criteria [24], WC $>80 \mathrm{~cm}$ according to International Diabetes Federation (IDF) criteria among the study participants $[13,25]$.

\section{Ethical approval}

Ethical approval for the study (IRB approval) was obtained from the University of Maryland, College Park, and the Ministry of Health - Jeddah Health Affairs Directorate in Saudi Arabia. Prior to the interview, each woman was asked to read and sign a consent form, which stated the purpose of the study, that participation was voluntary, and that women's responses were to be kept confidential.

\section{Statistical Analysis}

All analyses were based on the complex sampling design using the SPSS Complex Samples Software (Version 23.0). The anticipated effects of the complex sample design were accounted for as well through a "design effect" adjustment to the sample size. The Taylor Series Linearization (TLS) method was used for variance estimation of non-linear statistics. Design variables for variance estimation included a sampling error stratum (SEST) and sampling error computing unit (SECU), as well as a sample weight. Descriptive statistics (using the SPSS subprogram CSDESCRIPTIVES) were used to describe the prevalence of obesity and the characteristics of the study population and its mean, standard error, frequency, and percentage. The anthropometric variables included height, weight, WC and BMI. Chi-square tests (using the SPSS subprogram CSTABULATE) were used to examine the statistical significance and extent of associations between the two categorical variables, while General Linear Model (using the SPSS subprogram CSGLM) was used to conduct the Independent Samples t-test to examine the statistical significance and extent of associations between those continuous variables. Significance was defined as p value $<.05$.

\section{Results}

\section{Socio-Demographic Characteristics, Physical Activity, and Lifestyle Factors of Study Participants}

The mean \pm SE age of study participants was $30.27 \pm 0.74$ years, and the majority (51.4\%) of the women were in the age group of 20-35 years, 64\% were married, 53.4\% were housewives. Moreover, 54.2\% had more than a high school diploma, and belonged to low $(39.1 \%)$ or middle $(52.8 \%)$ income levels. One-third of married women (31\%) had 4 children or more, and $94 \%$ of them had breastfed their children for seven months or greater. One-third (33.0\%) of the women reported having health problems (in particular, chronic conditions, such as type 2-diabetes, hypertension, cardiovascular disease, hypercholesterolemia, and hypertriglyceridemia), while, $84.7 \%$ of women reported a family health history for chronic diseases (blood relatives).

Regarding the PA levels (Table 1), about $68 \%$ of the women were physically active, and $87.6 \%$ of them spent their leisure time in sedentary behaviors (e.g., watching TV, using phones or computers), and sat nearly 3 hours during the day. Moreover, their average amount of sleep per night was $6.5 \pm 0.1$ hours, and $54.1 \%$ of them had taken afternoon naps (siesta).

The results showed $57.3 \%$ of the women preferred to do the PA outdoors during anytime of the year. More than half of the women (50.5\%) preferred to exercise in public, while $45.1 \%$ of the women preferred to exercise at home. The main reasons given by women for engaging in PA were: because of health benefits (73.8\%) or weight reduction (47.6\%). Additionally, $85.2 \%$ of the women said that hot weather prevented them from exercising or walking outdoors, while $75 \%$ of the women said that it is not safe to walk in their neighborhoods. There were a high proportion of women (85.8\%) who needed permission from their families to practice PA outside the home, while $41.6 \%$ of them found that wearing the Abaya made them feel uncomfortable about walking in public areas. 


\begin{tabular}{|c|c|c|c|}
\hline Variables & $n$ & $\%$ & Mean \pm SE \\
\hline Sleeping hours per night $($ mean \pm SE) & & & $6.5 \pm 0.1$ \\
\hline Sitting hours per day (mean \pm SE) & & & $2.9 \pm 0.1$ \\
\hline Taking a nap & 220 & 54.1 & \\
\hline Using cars for transportation & 404 & 99.2 & \\
\hline \multicolumn{4}{|l|}{ Leisure time activities } \\
\hline Walking & 18 & 4.4 & \\
\hline Sport & 8 & 1.9 & \\
\hline Shopping & 27 & 6.4 & \\
\hline Dancing & 24 & 5.8 & \\
\hline $\begin{array}{c}\text { Watching TV/listening to music /using a computer or } \\
\text { phone/ reading }\end{array}$ & 358 & 87.8 & \\
\hline Other & 44 & 10.7 & \\
\hline Having maid & 115 & 28.3 & \\
\hline Physical activity (MET min/week) & & & $953.1 \pm 62.9$ \\
\hline \multicolumn{4}{|l|}{ Physical activity levels } \\
\hline Physical inactive & 131 & 32.0 & \\
\hline Physical active & 277 & 68 & \\
\hline Waking 150 mints or more /week & 118 & 29 & \\
\hline Physically inactive with family history of obesity & 31 & 52 & \\
\hline Physically active without family history of obesity & 248 & 71.4 & \\
\hline \multicolumn{4}{|l|}{ Prefer time (year) to do physical activity outdoors ${ }^{\mathrm{a}}$} \\
\hline Fall & 6 & 1.5 & \\
\hline Winter & 104 & 25.4 & \\
\hline Spring & 57 & 14 & \\
\hline Summer & 26 & 6.3 & \\
\hline Any time & 234 & 57.3 & \\
\hline \multicolumn{4}{|l|}{ Place prefer to do physical activity ${ }^{a}$} \\
\hline At home & 184 & 45.1 & \\
\hline At gym & 82 & 20.1 & \\
\hline School & 6 & 1.7 & \\
\hline At work & 4 & 1.0 & \\
\hline In public physical activity facility & 206 & 50.5 & \\
\hline Around inside malls & 23 & 9.5 & \\
\hline \multicolumn{4}{|l|}{ Main reasons for doing regular physical activity ${ }^{\mathrm{a}}$} \\
\hline Health benefits & 301 & 73.8 & \\
\hline To lose weight & 194 & 47.6 & \\
\hline Recreation & 51 & 12.4 & \\
\hline Media influence their body image & 291 & 71.4 & \\
\hline Celebrity stars influence on women body image & 229 & 56.0 & \\
\hline Having exercise equipment at home & 145 & 36.5 & \\
\hline Too expensive to purchase a exercise equipment & 317 & 47.6 & \\
\hline Too expensive to join a gym & 298 & 75.3 & \\
\hline Not safe to walk in neighborhood & 306 & 75 & \\
\hline $\begin{array}{l}\text { Permeation need family to practice physical activity } \\
\text { outside the home }\end{array}$ & 350 & 85.8 & \\
\hline $\begin{array}{l}\text { Wearing the Abaya makes me feel uncomfortable to walk } \\
\text { outside }\end{array}$ & 169 & 41.6 & \\
\hline Too hot to exercise outdoors & 348 & 85.2 & \\
\hline
\end{tabular}

SE: Standard Error; MET: Metabolic Equivalent Task

${ }^{a}$ Respondents can select more than one answer or choice (multiple response items)

Table 1: Physical activity and lifestyle factors for non-pregnant Saudi women of reproductive age residing in Jeddah city, Saudi Arabia $(n=408)$ 
There was a significant difference between active and inactive women according to their family history of obesity ( $\mathrm{p}<0.006)$. Nearly $52 \%$ of the women who had family history of obesity were physically inactive, while $71.4 \%$ of the women who did not have family history of obesity were physically active. Women with family history of obesity were 0.4 times more likely to be physical inactive as compared to women with family history of obesity (95\% CI: $0.2-0.7$ ). No statistically significant association was detected between age groups, marital status, education levels, occupation status, or income status and PA levels.

\section{Prevalence of General Obesity (BMI) Among Study Population}

Mean height and weight of participants were $156.5 \pm 0.41 \mathrm{~cm}$ and $67.7 \pm 1.3 \mathrm{~kg}$, respectively (Table 2). The results show that mean BMI increased from $24.0 \mathrm{~kg} / \mathrm{m}^{2}$ in adolescent women (15-18years) to $30.6 \mathrm{~kg} / \mathrm{m}^{2}$ in middle-age women (36-49years) (Table 3 ). Based on the WHO BMI classifications [19], the prevalence of both overweight and obesity was extraordinarily high among Saudi women who attended JPHCCs. Nearly 63\% of women included in this study population between 15 and 49 years of age were overweight or obese.

\begin{tabular}{|c|c|c|c|}
\hline $\begin{array}{l}\text { Anthropometric } \\
\text { characteristics }\end{array}$ & $\mathbf{n}$ & $\%^{a}$ & Mean \pm SE \\
\hline Height $(\mathrm{cm})$ & & & $156.5 \pm 0.4$ \\
\hline Weight (kg) & & & $67.7 \pm 1.3$ \\
\hline \multicolumn{4}{|l|}{ BMI $\left(\mathrm{kg} / \mathrm{m}^{2}\right)$ WHO cutoff } \\
\hline Underweight $(<18.5)$ & 41 & 10.1 & \\
\hline Normal (18.5-24.9) & 108 & 26.6 & \\
\hline Overweight (25-29.9) & 121 & 29.5 & \\
\hline Obese $(\geq 30)$ & 138 & 33.8 & \\
\hline $\mathrm{WC}(\mathrm{cm})$ & & & $80.4 \pm 1.1$ \\
\hline \multicolumn{4}{|l|}{ WC $(\mathrm{cm})$ WHO cutoff } \\
\hline Normal $(<88)$ & 306 & 75.0 & \\
\hline Health Risk ( $\geq 88)$ & 102 & 25.1 & \\
\hline \multicolumn{4}{|l|}{ WC $(\mathrm{cm})$ IDF cutoff } \\
\hline Normal $(<80)$ & 216 & 53.0 & \\
\hline Health Risk $(\geq 80)$ & 192 & 47.1 & \\
\hline
\end{tabular}

SE: Standard Error; kg: kilogram; m²: meter square; cm: centimeter; BMI: Body Mass Index; WHO: World Health Organization; WC: Waist Circumference; IDF: International Diabetes Federation

aPercentage may not total to $100 \%$ due to rounding

Table 2: Anthropometric characteristics of non-pregnant Saudi women of reproductive age residing in Jeddah city, Saudi Arabia $(n=408)$

\begin{tabular}{|c|c|c|c|}
\hline & $\begin{array}{l}\text { Adolescent }(=<19 \\
\text { years }) \text { Mean } \pm \text { SE }\end{array}$ & $\begin{array}{l}\text { Young women }(20-35 \\
\text { years) Mean } \pm \text { SE }\end{array}$ & $\begin{array}{c}\text { Middle-aged women } \\
(36-49 \text { years) Mean } \pm \mathrm{SE}\end{array}$ \\
\hline BMI & $24.0 \pm 1.3$ & $27.1 \pm 0.5$ & $30.6 \pm 0.5$ \\
\hline \multirow[t]{2}{*}{ WC } & $70.6 \pm 2.3$ & $78.2 \pm 1.0$ & $88.6 \pm 0.8$ \\
\hline & $n(\%)^{\mathrm{d}}$ & $n(\%)^{\mathrm{d}}$ & $n(\%)^{\mathrm{d}}$ \\
\hline Underweight (BMI <18.5) & $20(28.3)$ & $19(9.0)$ & $2(1.9)$ \\
\hline $\begin{array}{l}\text { Normal weight } \\
(\mathrm{BMI}=18.5-24.9)\end{array}$ & $25(35.8)$ & $66(31.7)$ & $17(13.1)$ \\
\hline Overweight $(\mathrm{BMI}=25-29.9)$ & $13(19.2)$ & $71(33.9)$ & $36(28.1)$ \\
\hline Obesity (BMI $\geq 30)$ & $12(16.7)$ & $53(25.4)$ & $73(56.9)$ \\
\hline $\begin{array}{l}\text { Abdominal obesity } \\
\qquad(W C \geq 88)^{b} \\
\text { Abdominal obesity } \\
\qquad(W C \geq 80)^{c}\end{array}$ & $\begin{array}{c}8(11.7) \\
16(22.6)\end{array}$ & $\begin{array}{l}37(17.7) \\
75(36.2)\end{array}$ & $\begin{array}{c}57(44.5) \\
100(78.5)\end{array}$ \\
\hline
\end{tabular}

BMI: Body mass index; WC: waist circumference

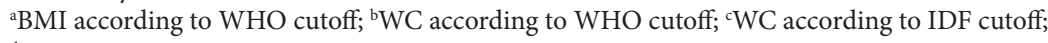
${ }^{\mathrm{d}}$ Percentage may not total to $100 \%$ due to rounding

Table 3: Prevalence of obesity by age according to $\mathrm{BMI}^{\mathrm{a}}$ and $\mathrm{WC}$ in non-pregnant Saudi women of reproduction age resident in Jeddah city, Saudi Arabia $(n=408)$

Overall, there were significant differences in overweight and obesity rates by age, marital status, parity, and having health conditions, while there were no significant differences in overweight and obesity rates in women by education levels, having maids, income levels, occupation status, and PA levels (Table 4). 


\begin{tabular}{|c|c|c|c|c|c|}
\hline \multirow[t]{2}{*}{ Variables } & \multirow[t]{2}{*}{$n$} & \multirow{2}{*}{$\begin{array}{c}\text { Total obesity } \\
\text { for whole } \\
\text { sample }(n=408)\end{array}$} & \multicolumn{2}{|c|}{$\begin{array}{c}\text { Non-obese \& obese prevalence } \\
\text { among each group }\end{array}$} & \multirow[t]{2}{*}{ p value ${ }^{\mathrm{b}}$} \\
\hline & & & Non-obese & Obese & \\
\hline $\begin{array}{c}\text { Age } \\
\text { Adolescent (15-19years) }\end{array}$ & 71 & $\begin{array}{c}n(\%)^{c} \\
25(9.7 \%)\end{array}$ & $\begin{array}{c}n(\%)^{\mathrm{c}} \\
46(64.1 \%)\end{array}$ & $\begin{array}{c}n(\%)^{c} \\
25(35.7)\end{array}$ & $<.001^{\star}$ \\
\hline Young women (20-35years) & 209 & $124(48.2 \%)$ & $85(40.7 \%)$ & $124(59.3 \%)$ & \\
\hline Middle age women (36-49years) & 128 & $109(42.1 \%)$ & $19(15 \%)$ & $109(85.0 \%)$ & \\
\hline $\begin{array}{l}\text { Education level } \\
\text { Less than high school }\end{array}$ & 125 & $84(32.5 \%)$ & $42(33.1 \%)$ & $84(66.9 \%)$ & 0.200 \\
\hline Completed high school & 99 & $65(125.3 \%)$ & $33(33.7 \%)$ & $65(66.3 \%)$ & \\
\hline More than high school & 184 & $109(42.3)$ & $75(40.8 \%)$ & $109(59.2 \%)$ & \\
\hline $\begin{array}{l}\text { Having maid } \\
\text { Yes }\end{array}$ & 115 & $73(28.4 \%)$ & $42(36.4 \%)$ & $73(63.6 \%)$ & 0.963 \\
\hline No & 293 & $185(71.5 \%)$ & $108(36.8 \%)$ & $185(63.2 \%)$ & \\
\hline $\begin{array}{c}\text { Income level } \\
\text { Low income }(<8,000 \text { SAR })\end{array}$ & 159 & $98(38.0 \%)$ & $61(38.4 \%)$ & $98(61.6 \%)$ & 0.815 \\
\hline Middle income (8000-18,000 SAR) & 216 & $137(52.2 \%)$ & $78(36.3 \%)$ & $137(63.7 \%)$ & \\
\hline High income (> 18,000 SAR) & 33 & $23(8.7 \%)$ & $11(13.6 \%)$ & $23(68.4 \%)$ & \\
\hline $\begin{array}{l}\text { Marital Status } \\
\text { Never married }\end{array}$ & 127 & $58(22.3 \%)$ & $69(54.7 \%)$ & $58(45.3 \%)$ & $0.01^{*}$ \\
\hline Married & 281 & $201(77.7 \%)$ & $80(28.6 \%)$ & $201(71.4 \%)$ & \\
\hline $\begin{array}{c}\text { Occupation status } \\
\text { Housewife }\end{array}$ & 218 & $137(53.0 \%)$ & $81(37.2 \%)$ & $137(66.2 \%)$ & 0.934 \\
\hline Employed & 87 & $57(21.9 \%)$ & $30(35.0 \%)$ & $57(65.0 \%)$ & \\
\hline Student & 103 & $65(25.1 \%)$ & $38(37.2 \%)$ & $65(62.8 \%)$ & \\
\hline $\begin{array}{c}\text { Parity (Live Births) groups } \\
\text { Nulliparous }\end{array}$ & 149 & $64(24.7 \%)$ & $85(57.2 \%)$ & $64(42.8 \%)$ & $<0.001^{\star}$ \\
\hline 1-2 children & 97 & $63(24.4 \%)$ & $34(34.9 \%)$ & $63(65.1 \%)$ & \\
\hline 3 children & 35 & $25(9.8 \%)$ & $10(28.8 \%)$ & $25(71.2 \%)$ & \\
\hline 4 and more children & 127 & $106(41.1 \%)$ & $21(16.2 \%)$ & $106(83.8 \%)$ & \\
\hline $\begin{array}{l}\text { Having Medical conditions } \\
\text { Yes }\end{array}$ & 135 & $122(47.2 \%)$ & $13(9.3 \%)$ & $122(90.7 \%)$ & $<0.001^{\star}$ \\
\hline No & 273 & $136(52.8 \%)$ & $137(50.2 \%)$ & $136(49.8 \%)$ & \\
\hline $\begin{array}{c}\text { Physical activity level } \\
\text { Inactive (<600 Met-min/week) }\end{array}$ & 131 & $85(33.2 \%)$ & $45(34.4 \%)$ & $85(65.6 \%)$ & 0.597 \\
\hline Active ( $\geq 600$ Met-min/week) & 277 & $172(66.8 \%)$ & $104(37.5 \%)$ & $172(62.2 \%)$ & \\
\hline
\end{tabular}

BMI: Body mass index; SAR: Saudi Arabian Riyal; MET: Metabolic Equivalent Task

${ }^{\mathrm{a}}$ Obesity at $\mathrm{BMI} \geq 25$ according to World Health Organization (WHO) criteria; ${ }^{\mathrm{b}} \mathrm{P}$ value for chi-square test of independence; cpercentage may not total to $100 \%$ due to rounding; ${ }^{*}$ significant $P<.05$

Table 4: The prevalence of general obesity (BMI) according to socio-demographic characteristics among non-pregnant Saudi women of reproduction age resident in Jeddah city, Saudi Arabia $(n=408)$

\section{Prevalence of Abdominal Obesity (WC) Among Study Population}

The mean WC, a measure of abdominal obesity of the entire study population was $80.4 \pm 1.1 \mathrm{~cm}$ (Table 2). The results show that mean WC increased from $70.6 \mathrm{~cm}$ in adolescent women (15-19 years) to $88.6 \mathrm{~cm}$ in middle-aged women (36-49 years) (Table 3). Using the WC as an indicator of abdominal obesity, $25.1 \%$ of women were abdominally obese based on WHO criteria (WC $\geq$ $88 \mathrm{~cm})$, and $47.1 \%$ were abdominally obese based on WC IDF criteria $(\mathrm{WC} \geq 80 \mathrm{~cm})$ (Table 2). Based on the WHO WC cut-off (Table 5), there were significant differences in abdominal obesity rates in women by age, parity, and having health conditions (in particular chronic diseases), while there were no significant differences in overweight and obesity rates in women by marital status, education levels, having housemaids, income levels, and occupation status, and PA level.

\section{Association of Family History of Chronic Diseases with General and Abdominal Obesity}

Table 6 displays the frequencies and percentages of family health history of chronic diseases (blood relatives) with general and abdominal obesity in study participants. A total of $346(84.7 \%)$ out of 408 women reported a positive family history for chronic diseases. Overall, there were significant differences between the women who had family health history of chronic diseases and those who did not, based on a comparison using BMI levels (classified into obese or not obese) $(\mathrm{p}<0.002)$, mean WC $(\mathrm{p}<0.001)$, 
and abdominal obesity with $\mathrm{WC} \geq 80 \mathrm{~cm}(\mathrm{p}<0.004)$ or $88 \mathrm{~cm}$ cut-off points $(\mathrm{p}<0.002)$, and personal history of chronic diseases $(\mathrm{p}<0.005)$ (Table 6). The mean WC for the women with a positive family history chronic diseases were above $82 \mathrm{~cm}$ across all family health conditions they reported (Table 8).

\begin{tabular}{|c|c|c|c|c|c|}
\hline Variables & $n$ & WC Mean \pm SE & $\begin{array}{l}\text { Total Abdom- } \\
\text { inal obesity } \\
\begin{array}{c}(n=408) \\
n(\%)^{b}\end{array}\end{array}$ & $\begin{array}{c}\text { Abdominal } \\
\text { obesity } \\
\text { among/group } \\
n(\%)^{b}\end{array}$ & p value ${ }^{c}$ \\
\hline $\begin{array}{c}\text { Age } \\
\text { Adolescent (15-19years) }\end{array}$ & 71 & $70.6 \pm 2.3$ & $8(8.1)$ & $8(11.7)$ & $<.001^{\star}$ \\
\hline Young women (20-35years) & 209 & $78.2 \pm 01.0$ & $37(36.3)$ & $37(17.7)$ & \\
\hline $\begin{array}{l}\text { Middle age women } \\
\quad(36-49 \text { years })\end{array}$ & 128 & $88.6 \pm 0.8$ & $57(55.6)$ & $57(44.5)$ & \\
\hline $\begin{array}{c}\text { Education level } \\
\text { Less than high school }\end{array}$ & 125 & $82.0 \pm 1.7$ & $39(38)$ & $39(31)$ & 0.085 \\
\hline Completed high school & 99 & $82.0 \pm 1.6$ & $31(30.4)$ & $31(31.6)$ & \\
\hline More than high school & 184 & $78.0 \pm 1.0$ & $32(31.6)$ & $32(17.5)$ & \\
\hline $\begin{array}{c}\text { Having maid } \\
\text { Yes }\end{array}$ & 115 & $80.7 \pm 1.8$ & $31(30.6)$ & $31(27.1)$ & 0.541 \\
\hline No & 293 & $79.9 \pm 1.1$ & $71(69.4)$ & $71(24.3)$ & \\
\hline $\begin{array}{c}\text { Income level } \\
\text { Low income }(<8,000 \mathrm{SAR})\end{array}$ & 159 & $80.7 \pm 1.1$ & $44(42.8)$ & $44(27.5)$ & 0.394 \\
\hline $\begin{array}{l}\text { Middle income (8000- } \\
18,000 \text { SAR) }\end{array}$ & 216 & $80.3 \pm 1.5$ & $53(52.0)$ & $53(24.7)$ & \\
\hline $\begin{array}{c}\text { High income (>18,000 } \\
\text { SAR) }\end{array}$ & 33 & $76.7 \pm 2.7$ & $5(5.1)$ & $5(15.9)$ & \\
\hline $\begin{array}{c}\text { Occupation status } \\
\text { Housewife }\end{array}$ & 218 & $81.4 \pm 1.35$ & $63(61.4)$ & $63(28.9)$ & 0.184 \\
\hline Employed & 87 & $79.0 \pm 1.7$ & $191(19)$ & $191(22.3)$ & \\
\hline Student & 103 & $78.6 \pm 1.5$ & $20(19.6)$ & $20(19.5)$ & \\
\hline $\begin{array}{l}\text { Marital Status } \\
\text { Never married }\end{array}$ & 127 & $74.4 \pm 2.4$ & $24(23.5)$ & $24(19)$ & 0.183 \\
\hline Married & 281 & $82.7 \pm 0.8$ & $78(76.5)$ & $78(27.8)$ & \\
\hline $\begin{array}{c}\text { Parity (Live Births) groups } \\
\text { Nulliparous }\end{array}$ & 149 & $73.4 \pm 1.9$ & $24(23.4)$ & $24(16.1)$ & $0.015^{\star}$ \\
\hline 1-2 children & 97 & $80.4 \pm 1.5$ & $18(18)$ & $18(19)$ & \\
\hline 3 children & 35 & $82.4 \pm 1.9$ & $11(10.7)$ & $11(30.7)$ & \\
\hline 4 and more children & 127 & $87.4 \pm 0.9$ & $49(47.9)$ & $49(38.8)$ & \\
\hline $\begin{array}{c}\text { Having Medical conditions } \\
\text { Yes }\end{array}$ & 135 & $90.7 \pm 1.3$ & $74(72.6)$ & $74(55.3)$ & $<0.001^{*}$ \\
\hline No & 273 & $75.0 \pm 0.7$ & $28(27.4)$ & $28(10.3)$ & \\
\hline $\begin{array}{c}\text { Physical activity level } \\
\text { Inactive }(<600 \text { Met-min/ } \\
\text { week })\end{array}$ & 131 & $82.0 \pm 1.4$ & $38(37.5)$ & $38(29.4)$ & 0.212 \\
\hline $\begin{array}{c}\text { Active ( } \geq 600 \mathrm{Met}-\mathrm{min} / \\
\text { week) }\end{array}$ & 277 & $79.3 \pm 1.2$ & $64(62.5)$ & $64(23.0)$ & \\
\hline
\end{tabular}

WC: Waist Circumference; SE: Standard Error; MET: Metabolic Equivalent Task

${ }^{a}$ Abdominal obesity, WC $\geq 88$ according to WHO criteria; ${ }^{b}$ mean \pm standard error; ${ }^{c}$ chi-square test of independence; *significant $\mathrm{p}=<.05$

Table 5: The prevalence of abdominal obesity according to socio-demographic characteristics among non-pregnant Saudi women of reproduction age resident in Jeddah city, Saudi Arabia $(n=408)$

\section{Association of Using Exercise Equipment at Home with General and Abdominal Obesity}

Table 7 displays percentages of exercise equipment use with general and abdominal obesity in women who had exercise equipment. The data indicated that $35.5 \%$ of women had exercise equipment, and $61.4 \%$ of those women were using it. The analysis revealed that there was no significant difference between the women who used the exercise equipment and those who had the exercise equipment but did not use it, based on comparisons using BMI level (obese or not obese), abdominal obesity, and mean WC, $\mathrm{p}<0.406, \mathrm{p}<0.463$, and $\mathrm{p}<0.283$, respectively. Furthermore, there were no significant differences between the women who used exercise equipment and who did not, based on their age grouping $(\mathrm{p}<0.246)$. 


\begin{tabular}{|c|c|c|c|}
\hline \multicolumn{3}{|c|}{ Family Health History of Chronic Diseases } & \multirow[b]{2}{*}{ p value } \\
\hline & $\begin{array}{l}\text { Yes } \\
n(\%)^{\mathrm{a}}\end{array}$ & $\begin{array}{l}\text { No } \\
n(\%)^{\mathrm{a}}\end{array}$ & \\
\hline Having family history of chronic disease & $346(84.7)$ & $62(15.3)$ & $<.001^{\mathrm{d}^{*}}$ \\
\hline \multicolumn{4}{|l|}{ BMI (WHO cut-offs) } \\
\hline Non-obese $(\mathrm{BMI}<25)(n$ 150) & $111(74.0)$ & $39(26.0)$ & $.002^{e^{*}}$ \\
\hline Obese (BMI $\geq 25)(n$ 258) & $235(91.0)$ & $23(9.0)$ & \\
\hline \multicolumn{4}{|l|}{ Abdominal obesity (WC $\geq 88)^{b}$} \\
\hline Non-obese $(n 62)$ & $248(81.1)$ & $58(18.9)$ & $.004^{e^{*}}$ \\
\hline Obese ( $n$ 346) & $97(95.5)$ & $5(4.4)$ & \\
\hline \multicolumn{4}{|l|}{ Abdominal obesity $(\mathrm{WC} \geq 80)^{c}$} \\
\hline Non-obese $(n$ 216) & $167(77)$ & $49(23.0)$ & $.002^{e^{*}}$ \\
\hline Obese ( $n$ 192) & $179(93.4)$ & $13(6.6)$ & \\
\hline $\begin{array}{c}\text { Medical conditions (chronic diseases) } \\
\text { No }(n 273)\end{array}$ & $218(80.0)$ & $55(20.0)$ & $.005^{e^{*}}$ \\
\hline \multirow[t]{2}{*}{ Yes $(n 135)$} & $127(94.4)$ & $8(5.6)$ & \\
\hline & Mean \pm SE & Mean \pm SE & \\
\hline WC $(\mathrm{cm})$ & $81.6 \pm 1.0$ & $72.1 \pm 1.9$ & $<.001^{\mathrm{e}}$ \\
\hline
\end{tabular}

BMI: Body Mass Index; WHO: World Health Organization; WC: Waist Circumference; SE: Standard Error aPercentage may not total to $100 \%$ due to rounding; ${ }^{b} \mathrm{WC}$ according to WHO cutoff; ' $\mathrm{WC}$ according to IDF cutoff; ${ }^{\mathrm{d}} \mathrm{p}$ value for chi-squared test for homogeneity; ${ }^{\mathrm{p}} \mathrm{p}$ value for chi-squared test of independence; ${ }^{\mathrm{f}} \mathrm{p}$ value for t-test for independent means; ${ }^{*}$ significant $\mathrm{p}=<.05$

Table 6: Association between family health history of chronic diseases and general and abdominal obesity among nonpregnant Saudi women of reproduction age resident in Jeddah city, Saudi Arabia $(n=408)$

\begin{tabular}{|c|c|c|c|}
\hline & \multicolumn{2}{|c|}{$\begin{array}{l}\text { Using exercise equip- } \\
\text { ment }\end{array}$} & \multirow{2}{*}{ p value } \\
\hline & $\begin{array}{l}\text { Yes } \\
n(\%)^{\mathrm{a}}\end{array}$ & $\begin{array}{l}\text { No } \\
n(\%)^{\mathrm{a}}\end{array}$ & \\
\hline Using exercise equipment & $89(61.4)$ & $56(38.6)$ & $0.019^{c^{*}}$ \\
\hline BMI (WHO cut-offs) & & & $0.406^{\mathrm{d}^{*}}$ \\
\hline Non-obese (BMI < 25) & $29(67.0)$ & $14(33.0)$ & \\
\hline Obese (BMI $\geq 25)$ & $61(59.7)$ & $41(40.3)$ & \\
\hline Abdominal obesity (WHO cut-offs) ${ }^{b}$ & & & $0.463^{\mathrm{d}^{*}}$ \\
\hline Non-obese $(\mathrm{WC}<88)$ & $65(64.7)$ & $36(35.3)$ & \\
\hline Obese (WC $\geq 88$ ) & $24(55.0)$ & $20(44.7)$ & \\
\hline $\begin{array}{c}\text { Age } \\
\text { Adolescent (15-19years) }\end{array}$ & $19(75.3)$ & $7(24.7)$ & $0.246^{\mathrm{d}^{*}}$ \\
\hline Young women (20-35years) & $48(63.3)$ & $28(36.7)$ & \\
\hline Middle age women (36-49years) & $22(51.0)$ & $21(49.0)$ & \\
\hline $\begin{array}{c}\text { Physical activity level } \\
\text { Inactivity (<600 Met-min/week) }\end{array}$ & $15(39.5)$ & $23(60.5)$ & $0.031^{\mathrm{d}^{*}}$ \\
\hline Active ( $\geq 600$ Met-min/week) & $75(70.0)$ & $32(30.1)$ & \\
\hline \multicolumn{4}{|l|}{$\begin{array}{l}\text { Times of using exercise equipment (at least } \\
30 \text { min or more)/week }\end{array}$} \\
\hline One time (30min/week) & $23(25.7)$ & $0.0 \%$ & \\
\hline \multirow[t]{2}{*}{ Two times and more (90 min or more/week) } & $66(74.3)$ & $0.0 \%$ & \\
\hline & Mean (SE) & Mean (SE) & \\
\hline $\mathrm{WC}(\mathrm{cm})$ & $81.3 \pm 1.9$ & $84.2 \pm 1.9$ & $0.283^{\mathrm{e}^{*}}$ \\
\hline
\end{tabular}

BMI: Body Mass Index; WHO: World Health Organization; WC: Waist Circumference; SE: Standard Error

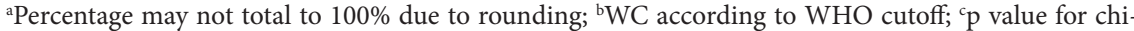
squared test for homogeneity; ${ }^{\mathrm{d}} \mathrm{p}$ value for chi-squared test of independence; ${ }^{\mathrm{p}} \mathrm{p}$ value for $\mathrm{t}$-test for independent means, ${ }^{*}$ significant $\mathrm{p}=<.05$

Table 7: Association between using exercise equipment and general and abdominal obesity among women who only have exercise equipment $(n=145)$ 


\begin{tabular}{|c|c|c|c|c|c|c|}
\hline Variables & $n(\%)$ & $\begin{array}{l}\text { WC Mean } \\
\text { (SE) }\end{array}$ & $\begin{array}{l}\text { Abdominal obesity } \\
\quad(\mathrm{WC} \geq 80)^{\mathrm{b}}\end{array}$ & $\begin{array}{l}\text { Abdominal obe- } \\
\text { sity }(\mathrm{WC} \geq 88)^{\mathrm{c}}\end{array}$ & $\begin{array}{l}\text { Non-obese } \\
(\mathrm{BMI}<25)\end{array}$ & 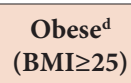 \\
\hline & & & $n(\%)$ & $n(\%)$ & $n(\%)$ & $n(\%)$ \\
\hline $\begin{array}{c}\text { Family history of chronic diseases }^{e} \\
\text { No }\end{array}$ & $62(15.3)$ & $72.1(1.9)$ & $13(20.4)$ & $4(7.2)$ & $39(62.4)$ & $23(37.6)$ \\
\hline Yes & $346(84.7)$ & $81.6(1.0)$ & $179(52.0)$ & $98(28.3)$ & $111(32.1)$ & $235(67.9)$ \\
\hline Obesity & $60(14.7)$ & $89.7(2.2)$ & $46(76.9)$ & $30(50.7)$ & $9(15.1)$ & $51(84.8)$ \\
\hline Diabetes & $277(68.0)$ & $82.6(1.3)$ & $151(54.5)$ & $85(30.6)$ & $81(29.4)$ & $196(70.6)$ \\
\hline Hypertension & $203(49.7)$ & $83.3(1.2)$ & $117(57.6)$ & $72(35.6)$ & $61(29.9)$ & $142(70.1)$ \\
\hline Cardiovascular Disease & $45(11.1)$ & $86.1(2.2)$ & $30(66.6)$ & $16(36.2)$ & $7(15.1)$ & $38(85.0)$ \\
\hline High Cholesterol Level & $44(10.7)$ & $85.5(1.8)$ & $29(66.0)$ & $15(35.3)$ & $13(29.1)$ & $31(71.0)$ \\
\hline High Triglyceride Level & $6(1.6)$ & $89.8(6.8)$ & $4(62.1)$ & $3(43.2)$ & $0(0.0)$ & $6(100.0)$ \\
\hline $\begin{array}{c}\text { Medical conditions (chronic diseases) } \\
\text { No }\end{array}$ & $274(67.1)$ & $75.0(0.7)$ & $83(30.4)$ & $28(10.2)$ & $137(50.2)$ & $136(49.8)$ \\
\hline Yes & $134(32.9)$ & $90.7(1.3)$ & $109(81.0)$ & $74(55.3)$ & $13(9.3)$ & $122(90.7)$ \\
\hline Diabetes & $36(8.8)$ & $90.5(2.1)$ & $30(84.7)$ & $20(56.7)$ & $3(8.4)$ & $33(91.6)$ \\
\hline Hypertension & $34(8.2)$ & $91.9(2.9)$ & $25(73.0)$ & $19(56.1)$ & $3(7.7)$ & $31(92.3)$ \\
\hline Cardiovascular Disease & $3(0.7)$ & $86.4(6.5)$ & $3(72.3)$ & $2(63.6)$ & $1(36.4)$ & $2(63.6)$ \\
\hline High Cholesterol Level & $33(8.0)$ & $87.5(1.8)$ & $23(70.0)$ & $13(38.7)$ & $4(13.1)$ & $29(87.0)$ \\
\hline High Triglyceride Level & $7(1.7)$ & $90.2(7.3)$ & $4(56.6)$ & $3(46.0)$ & $2(24.0)$ & $5(76.0)$ \\
\hline
\end{tabular}

WC: waist circumference; BMI: Body mass index. SE: standard error

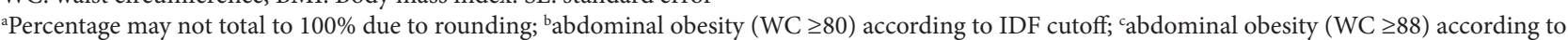
WHO criteria; 'general obesity (BMI $\geq 25$ ) according to WHO criteria; ${ }^{e}$ respondents can select more than one answer or choice (multiple response items) Table 8: Differences in the obesity percentagesa and mean WC among non-pregnant Saudi women of reproductive age residing in Jeddah city, Saudi Arabia when comparing those with and without family or personal history of chronic disease according to general and abdominal obesity $(n=408)$

\section{Discussion}

\section{Prevalence of General Obesity Measured by BMI}

Based on the WHO BMI classifications, rates of overweight and general obesity among non-pregnant Saudi women of reproductive age (15-49 years) seen in PHCCs in Jeddah City (29.5\% and 33.8\%, respectively) are higher than those obtained from a national representative data collected over 10 years among all U.S. women of reproductive age (15-49 years) (24.6\% and $30.8 \%$, respectively) [26]. Comparing our rates with the Al-malik study [27], we find the following: in the local study conducted in Riyadh City among Saudi women of reproductive age (16-45 years old), the prevalence of overweight in the Al-malik study was slightly higher (31.5\%) than our rate (29.5\%), but its prevalence of obesity was lower $(20.86 \%)$ than our rate $(33.8 \%)$. These differences in the obesity rates are reasonable, because the women in the Al-malik study [27] were younger than the women in our study (mean age 26 and 30 years, respectively), and the majority of them were under 30 years old ( $76 \%$ and $52.6 \%$, respectively), and were unmarried ( $51.2 \%$ and $31 \%$, respectively).

For further comparison, we reviewed three previous Saudi National Surveys that determined the prevalence of overweight and obesity in women using the WHO cut-offs. Our study rates are comparable to those ( $28 \%$ and $33.5 \%$, respectively) reported by the latest National Saudi Health Information Survey (SHIS) (aged $\geq 16$ years) [3], lower than those reported by Al-Nozha et al. [28] (31.8\% and 44\%, respectively), and higher than those estimated by Al-Othaimeen et al. [29] (28.4\% and 23.4\%, respectively) in 3070 year old Saudi women. According to these surveys, the rates of obesity among Saudi women declined about 10.2\% from 2005 to 2013, and this drop may have resulted from the difference in age groups, or due to the public health program implementations of the Saudi Ministry of Health (SMOM). These programs have focused on awareness and behavioral changes [3]. However, it is too early to determine whether the decline seen in this study is due to chance or really due to the changes in health behavior. Additionally, compared with other national surveys in the Middle Eastern countries, which used WHO diagnostic criteria, prevalence of general obesity in our study was lower than in Kuwait (53.0\%), Bahrain (40.3\%), Turkey (44\%) countries [30-32], but higher than in Oman (22.3\%), Jordan (27.4\%), Lebanon (25.9\%), and Iran (25.2\%) countries [33-36]. This worldwide variation in the prevalence of general obesity could not simply be due to ethnicity, and may involve a wide range of genetic, sociodemographic, environmental, and cultural factors [37]. Ethnicity is used to categorize populations on the basis of cultural characteristics such as shared ancestry, language, religious traditions, dietary preferences, and history. Although ethnic groups can share a range of phenotypic characteristics due to their shared ancestry, the term is typically used to highlight cultural and social characteristics instead of biological ones [38].

\section{Prevalence of Abdominal Obesity Measured by WC}

In the present study, the mean WC for women $(80 \pm 1.12 \mathrm{~cm})$ was smaller than the mean WC $(90.5 \mathrm{~cm})$ of American women of reproductive age (15-49 years old) who participated in the national representative data collected over 10 years [26]. Compared 
to some Saudi studies that measured the mean WC of women, we found our mean WC was smaller than other reported studies. The mean WC was $84.2 \mathrm{~cm}$ among non-pregnant Saudi women aged 18-60 years in Abha City [39], and a Saudi national representative household survey found that the mean WC was $82.9 \pm 1.43 \mathrm{~cm}$ among 2,416 women, age between 15-64 years old [40]. Unfortunately, however, prevalence of abdominal obesity (WC) was not reported in this survey. These differences in the mean WC might be attributed to the women in our study belonged to a young age group and the majority of them were under 30 years old (52.6\%).

Prevalence of abdominal obesity using the WHO cut-off (WC $\geq 88 \mathrm{~cm}$ ) was $25.1 \%$ among the study population, which was lower than the prevalence of abdominal obesity in non-pregnant American women (48.8\%) of reproductive age (representative data collected over 10 years). In Saudi Arabia, only a limited number of studies have examined the prevalence of abdominal obesity among Saudi women, specifically those of a reproductive age. Comparing our rate of abdominal obesity using the WC cut-off point $(\geq 88 \mathrm{~cm})$ to previous two national surveys (30-70 years) [41,42], we found that our rate was lower than their rates, $66.1 \%$ and $55.2 \%$ respectively. In addition, our rate was lower than that of some other Middle East countries, such as Oman $(\geq 20$ years of age) and Iran ( $\geq 15-65$ years of age) $(44.3 \%$ and $53.2 \%$ respectively) $[43,44]$. Using two different WC cut-offs, the abdominal obesity prevalence among Kuwaiti adult women ( $\geq 20$ years) obtained from the National Nutrition Survey was higher than our rates; the prevalence of abdominal obesity was ( $59.7 \%$ and $25.1 \%$, respectively) according to WHO criteria (WC $\geq 88 \mathrm{~cm})$, and the prevalence of abdominal obesity was (78.1\% and $47.1 \%)$ according to IDF criteria (WC $\geq 80 \mathrm{~cm})$ [45]. These observed variations in the prevalence of abdominal obesity could be contributing to the differences in sample characteristics such as age or sociodemographic variables. In the current study, it seems that women are young (15-49 years old), and a high proportion of them are under the age of 35 years (68.7\%), unmarried (31\%), and have not had children (36\%). Therefore, these characteristics may lead to lower rates of abdominal obesity among them, since the abdominal fat is lower at younger ages, and increases with age, marriage and having children.

\section{Association of Family History of Chronic Diseases with General and Abdominal Obesity}

Family history of chronic disease offers valuable genomic information and environmental risk factors [46]. Family members or biological relatives share their genetic information as well their behavior, belief, lifestyle, culture, and physical environment [47]. The current study demonstrated that women with a positive history of chronic disease had significantly higher rates of both types of obesity and greater mean WC compared to women who had a negative family history of chronic diseases. The increased prevalence risk of obesity observed among women who reported a positive family history of chronic diseases is in accord with the results from other studies [48-53]. Therefore, identification and awareness of the family history of chronic disease will serve as a practical and useful approach for public health and preventive health conditions. Also, promotion of healthy behaviors such as regular PA, nutrition education, and routine health checkups, along with keeping track of family health history to continue monitoring the obesity and other health condition risks, would be desirable for women to lower the risks of developing common chronic diseases in the future [52,54].

In the current study, using different cut-off values of WC (WHO and IDF WC cut-offs) yielded different conclusions regarding the diagnosis of abdominal obesity and its related diseases. According to the IDF WC cut-off point (WC $\geq 80 \mathrm{~cm}$ ), this study found that all women who had a family history of chronic disease or had one or more health conditions were at high risk of obesity-related diseases. These findings support previous studies that suggest that ethnic variation among populations from different countries might require different anthropometric measurement cut-off points to diagnose obesity and its related diseases [13,25]. This decision was made because studies had confirmed that disease risk in Asian people was high at WC cut-off points below those defined in European populations [55-57]. Therefore, further research is essential to determine ethnic-specific anthropometric cutoff points for the Saudi Arabia population, which may potentially be beneficial in correctly identifying individuals at high risk for developing obesity and its related diseases.

\section{PA and Lifestyle Behaviors}

PA is an important component of a healthy lifestyle, and has been described as an essential factor in managing many health conditions and combatting the obesity epidemic [58,59]. By analyzing the National Health and Nutrition Examination Survey (NHANES) data from the last 20 years (1988-2010), researchers from Stanford University discovered a sharp decrease in PA and an increase in average BMI and WC, while caloric intake remained steady [60]. They identified significant associations between the reported level of leisure-time PA in the U.S. population and all measures of obesity, but they did not find any evidence that average daily caloric intake increased over the last two decades.

By using the IPAQ short-form instrument, the current study found that physical inactivity among the study sample was $31.2 \%$. These findings confirmed, and were consistent with, the high prevalence of physical inactivity (34.3\%) (Assessed by IPAQ) among Saudi women reported by Al-Hazzaa [12]. In the Al-Hazzaa study and our study, there were low proportions (28.5\% vs. $29 \%$ ) of women meeting the USDA PA guidelines for adults (a minimum of 150 minutes/week of moderate activity for weight management) [11].

A number of cross-sectional and longitudinal studies have shown an inverse association between PA and obesity, suggesting 
that physical inactivity may precede the development of obesity [61-63]. However, in current study, there were no significant associations between PA and either type of obesity and mean WC among the study group. These findings were consistent with the finding of the latest Saudi National Health Survey, which found no association between PA and general obesity among Saudi women aged 15 years and older. Possible reasons for inconsistent results about the relationship between PA and obesity are as follows. First, most of the women in our study reported being physically active (68\%). Second, some studies deal with PA as a continuous variable (Metabolic Equivalent Task [MET] minutes per week) and others treat it as a categorical variable (active and inactive level). Third, different methods were used to assess PA levels in the studies. Finally, the possibility of measurement error in the physical activity assessment.

Obese family members create an obesogenic household and reflect behaviors in the family that may lead to sedentary lifestyles [64-66]. Our study indicates that family history of obesity was significantly associated with increased sedentary behaviors among women with a history of obesity. This finding is in agreement with previous studies. A cross-sectional study was recently conducted at the Aga Khan University Hospital (AKUH), Karachi, Pakistan to assess the prevalence of physical inactivity in 350 obese adults (mean age 41 years, $53.4 \%$ women). The study found that subjects with a positive family history of obesity were 3.5 times more at risk of being physically inactive relative to those without a family history of obesity [67].

This study indicated some factors that make PA in public or outdoors a difficult choice for women, including requiring a guardian for commuting, needing family permission to practice PA outside the home, wearing an Abaya, hot weather, the cost of a gym, and feeling unsafe walking in the neighborhood. Therefore, we found a high proportion of the women (45.1\%) preferred to do PA at home. Overall, 35.6\% of the women had exercise equipment at home, and a high percentage of them (61.4\%) used it. As might be expected, women who used the exercise equipment were significantly more physically active $(p<0.031)$ than the women who had the equipment but did not use it. The majority of those who had used the exercise equipment at home used it two times or more per week for at least 90 minutes or more. This finding may reflect the effect of regular exercise on increased energy expenditure, which may improve long-term weight loss outcomes [58].

Regarding high rates of obesity $70 \%$ among the women who had exercise equipment, we could not find any significant differences between the women who used the exercise equipment and the women who had it but did not use it, based on all measures of obesity (BMI and WC). However, the mean WC for the women who used the exercise equipment $81.3 \pm 1.9 \mathrm{~cm}$ was smaller than the mean WC $84.2 \pm 1.9 \mathrm{~cm}$ for women who had equipment but did not use it. A randomized controlled study was conducted to determine the effects of different amounts and intensities of exercise training among 120 overweight men and women (aged 4065 years) in the City of Durham, North Carolina, USA. The results suggested that eight months of both low (114 min/week) and high (175 min/week) amounts of PA were associated with significant reductions in WC in the study group [68]. These findings highlight the association between the increasing PA and the reduction in abdominal fat $[69,70]$. Therefore, we greatly recommend that women should increase the weekly workout intensity level and duration to get a significant impact of weight loss.

The study had a few limitations. First, the cross-sectional nature of the study would not allow for cause-effect relationships to be established between socio-demographic factors and obesity. Second, the questionnaire assessments of PA are subject to recall bias, and the self-reported PA did not provide accurate estimates of absolute amounts of activity (Metabolic Equivalent Task minutes per week). A third limitation was that the results of the study could only be generalized to non-pregnant Saudi women of reproductive age (15-49 years) who obtained services at PHCCs in Jeddah City, and were not applicable to all Saudi women living in Jeddah or other cities in Saudi Arabia. Therefore, replication of this study using representative sample of Saudi population (National study) is highly suggested. The National study should investigate the abdominal obesity using WC beside the general obesity (BMI). This study may provide accurate data and result in population-specific recommendations that help develop appropriate and effective obesity prevention strategies for different groups of Saudi women.

\section{Conclusion}

The prevalence of general and abdominal obesity was remarkably high in Saudi women of reproductive age who obtained services at JPHCCs. Most important, our study general obesity rate is comparable to those reported by the latest National Saudi Health Information Survey (SHIS) among 16 years of age and older Saudi women [3], suggesting that our obesity rate could be also generalized to all non-pregnant Saudi women. Moreover, our study showed high levels of inactive lifestyle among the study population. Conservative Saudi society, cultural norms, and politics have a great impact on the women's PA level. Therefore, an intervention program to combat obesity is greatly needed, especially one that focuses on PA and mitigates social norms. Moreover, the study suggests that promotion of healthy behaviors such as regular PA, nutrition education, and routine health checkups, along with keeping track of family health history to continue monitoring obesity and other health condition risks, would be desirable for women to lower the risk of developing common chronic diseases in the future. We also observed that using different cut-off values for WC (WHO and IDF WC cut-offs) yielded different conclusions regarding the diagnosis of abdominal obesity and its related diseases. Thus, further research is essential to determine ethnic-specific anthropometric cut-off points for the Saudi Arabia population, which may potentially be beneficial in correctly identifying individuals at high risk for developing obesity and its related diseases. 


\section{Acknowledgment}

The authors wish to thank the Jeddah Primary Health Centers (JPHCCs) that provided us the opportunity to conduct research at their facilities.

\section{References}

1. Flegal KM, Carroll MD, Ogden CL, Curtin LR (2010) Prevalence and trends in obesity among US adults, 1999-2008. JAMA 303: 235-41.

2. Low S, Chin MC, Deurenberg-Yap M (2009) Review on Epidemic of obesity. Ann Acd Med Singapore 38: 57-9.

3. Memish ZA, El Bcheraoui C, Tuffaha M, Robinson M, Daoud F, et al. (2014) Obesity and associated factors Kingdom of Saudi Arabia, 2013. Prev Chronic Dis 11: E174.

4. Daoud F, El Bcheraoui C, Tuffaha M, AlMazroa MA, Al Saeedi M, et al. (2015) The health status of Saudi women: findings from a national survey. J Public Health (Oxf) doi: 10.1093/pubmed/fdv157.

5. Gunderson EP (2009) Childbearing and obesity in women: weight before, during, and after pregnancy. Obstet Gynecol Clin North Am 36: 317-32.

6. Hillemeier MM, Weisman CS, Chuang C, Downs DS, McCall-Hosenfeld J, et al. (2011) Transition to overweight or obesity among women of reproductive age. J Womens Health (Larchmt) 20: 703-10.

7. Ravensbergen HR, Lear SA, Claydon VE (2014) Waist circumference is the best index for obesity-related cardiovascular disease risk in individuals with spinal cord injury. J Neurotrauma 31: 292-300.

8. Coutinho T, Goel K, Corrêa de Sá D, Kragelund C, Kanaya AM, et al. (2011) Central obesity and survival in subjects with coronary artery disease: a systematic review of the literature and collaborative analysis with individual subject data. J Am Coll Cardiol 57: 1877-86.

9. Browning LM, Hsieh SD, Ashwell M. (2010) A systematic review of waist-to-height ratio as a screening tool for the prediction of cardiovascular disease and diabetes: 0.5 could be a suitable global boundary value. Nutr Res Rev 23: 247-69.

10. Shields M, Tremblay MS, Connor Gorber S, Janssen I (2012) Abdominal obesity and cardiovascular disease risk factors within body mass index categories. Health Rep 23: 7-15.

11. US Department of Agriculture and US Department of Health and Human Services (2010) Dietary Guidelines for Americans, USA.

12. Al-Hazzaa HM (2007) Health-enhancing physical activity among Saudi adults using the International Physical Activity Questionnaire (IPAQ). Public Health Nutr 10: 59-64.

13. Alberti KGMM, Eckel RH, Grundy SM, Zimmet PZ, Cleeman JI, et al. (2009) Harmonizing the metabolic syndrome: a joint interim statement of the International Diabetes Federation Task Force on Epidemiology and Prevention; National Heart, Lung, and Blood Institute; American Heart Association; World Heart Federation; International Atherosclerosis Society; and International Association for the Study of Obesity. Circulation 120: 1640-5.

14. Khashoggi RH, Madani KA, Ghaznawy HI, Ali MA (1994) Socioeconomic factors affecting the prevalence of obesity among female patients attending primary health centers in Jeddah, Saudi Arabia. Ecol Food Nutr 31: 277-83.

15. Arabic short version of International Physical Activity Questionnaire (2002) Stockholm, Karolinska Institutet, Sweden.

16. Craig CL, Marshall AL, Sjostrom M, Bauman AE, Booth ML, et al. (2003) International physical activity questionnaire: 12-country reliability and validity. Med Sci Sports Exerc 35: 1381-95.

17. Al-Eisa ES, Al-Sobayel HI (2012) Physical Activity and Health Beliefs among Saudi Women. J Nutr Metab 2012: 642187.

18. AboZaid HA, Farahat FM (2010) Physical activity profile among patients attending family medicine clinics in western Saudi Arabia. Saudi Med J 31: 428-33.

19. Al-Hazzaa HM (2004) The public health burden of physical inactivity in Saudi Arabia. J Family Community Med 11: 45-51.

20. International Physical Activity Questionnaire (2005) Guidelines for data processing and analysis of the International Physical Activity Questionnaire (IPAQ).

21. World Health Organization (2012) Global Database on Body Mass Index. BMI classification.

22. de Onis M, Onyango AW, Borghi E, Siyam A, Nishida C, et al. (2007) Development of a WHO growth reference for school-aged children and adolescents. Bull World Health Organ 85: 660-7.

23. World Health Organization (2010) Anthro plus for personal computers, version 3.3.2, 2011: Software for assessing growth and development of the world's children, Geneva.

24. World Health Organization (2011) Waist Circumference and Waist-Hip Ratio. Report of a WHO Consultation, Geneva.

25. Alberti KGMM, Zimmet P, Shaw J (2006) Metabolic syndrome-a new worldwide definition. A Consensus Statement from the International Diabetes Federation. Diabet Med 23: 469-80.

26. Marcinkevage JA, Alverson CJ, Narayan KM, Kahn HS, Ruben J, et al. (2013) Race/ethnicity disparities in dysglycemia among U.S. women of childbearing age found mainly in the nonoverweight/nonobese. Diabetes Care 36: 3033-9.

27. Al-Malki JS, Al-Jaser MH, Warsy AS (2003) Overweight and obesity in Saudi females of childbearing age. Int J Obes Relat Metab Disord 27: 134-9.

28. Al-Nozha MM, Al-Mazrou YY, Al-Maatouq MA, Arafah MR, Khalil MZ, et al. (2005) Obesity in Saudi Arabia. Saudi Med J 26: 824-9.

29. Al-Othaimeen AI, Al-Nozha M, Osman AK (2007) Obesity: an emerging problem in Saudi Arabia. Analysis of data from the National Nutrition Survey. East Mediterr Health J 13: 441-8.

30. Ministry of Health (2007) Risk Factors for Non-communicable Diseases in Kuwait.

31. Ministry of Health (2010) National Non-communicable Diseases Risk Factors Survey 2007, Bahrain.

32. Erem C (2015) Prevalence of overweight and obesity in Turkey. IJC Metabolic \& Endocrine 8: 38-41.

33. Ministry of Health (2000) National Health Survey: Study of Lifestyle Risk Factors in Oman.

34. Al-Nsour M, Arbaji A (2014) Obesity and Related Factors among Jordanian Women of Reproductive Age Based on Three DHS Surveys, 2002-2012. DHS Working Papers No. 115. 2014. Rockville, Maryland, USA: ICF International, USA.

35. Chamieh MC, Moore HJ, Summerbell C, Tamim H, Sibai AM, et al. (2015) Diet, PA and socio-economic disparities of obesity in Lebanese adults: findings from a national study. BMC Public Health 15: 279. 
36. Janghorbani M, Amini M, Willett WC, Mehdi Gouya M, Delavari A, et al. (2007) First nationwide survey of prevalence of overweight, underweight, and abdominal obesity in Iranian adults. Obesity (Silver Spring) 15: 2797-808.

37. Wittchen H, Balkau B, Massien C, Richard A, Haffiner S, et al. (2006) International day for evaluation of abdominal obesity: rationale and design of a primary care study on the prevalence of abdominal obesity and associated factors in 63 countries. Eur Heart J 8: B26-33.

38. Caprio S, Daniels SR, Drewnowski A, Kaufman FR, Palinkas LA, et al. (2008) Influence of Race, Ethnicity, and Culture on Childhood Obesity: Implications for Prevention and Treatment: A consensus statement of Shaping America's Health and the Obesity Society. Diabetes Care 31: 2211-21.

39. Khalid ME (2007) The prevalence of abdominal obesity and its associated risk factors in married, non-pregnant women born and living in high altitude, southwestern, Saudi Arabia. Saudi Med J 28: 1875-80.

40. Ministry of Health (Saudi Arabia), World Health Organization (WHO) (2005) Saudi Arabia STEPS Noncommunicable Disease Risk Factors Survey $2004-2005$. 41. Al-Saif MA, Hakimb IA, Harrisc RB, Al-duwaihyd M, Al-Rubeaand K, et al. (2002) Prevalence and risk factors of obesity and overweight in adult Saudi population. Nutrition Research 22: 1243-52.

42. Al-Nozha M, Al-Khadra A, Arafah MR, Al-Maatouq MA, Khalil MZ, et al. (2005) Metabolic syndrome in Saudi Arabia. Saudi Med J $26: 1918-25$.

43. Al-Lawati JA, Mohammed AJ, Al-Hinai HQ, Jousilahti P (2003) Prevalence of the metabolic syndrome among Omani adults. Diabetes Care 26: 1781-5.

44. Veghari G, Sedaghat M, Banihashem S, Moharloei P, Angizeh A, et al. (2012) Trends in waist circumference and central obesity in adults, northern iran. Oman Med J 27: 50-3.

45. Al-Zenki S, Al-Omirah H, Al Hooti S, Al-Hamad N, Jackson RT, et al. (2012) High prevalence of metabolic syndrome among Kuwaiti adults--a wake-up call for public health intervention. Int J Environ Res Public Health 9: 1984-96.

46. Claassen L, Henneman L, Janssens AC, Wijdenes-Pijl M, Qureshi N, et al. (2010) Using family history information to promote healthy lifestyles and prevent diseases; a discussion of the evidence. BMC Public Health 10: 248.

47. Khanna N, Sharma RS, Sidhu RS (2011) A study of the basic and derived anthropometric indices among the healthy adults (20-30 years of age) of amritsar city (punjab) having family history of hypertension. Int J Biol Med Res 2: 743-6.

48. Issa LF (2015) Prevalence and risk factors of obesity and overweight among Taif University Students, Taif, Saudi Arabia. Int J Public Health 41: 98-106.

49. Al-Qauhiz NM (2010) Obesity among Saudi Female University Students: Dietary Habits and Health Behaviors. J Egypt Public Health Assoc 85: 45-59.

50. Erem C, Arslan C, Hacihasanoglu A, Deger O, Topbas M, et al. (2004) Prevalence of obesity and associated risk factors in a Turkish population (trabzon city, Turkey). Obes Res 12: 1117-27.

51. Suleiman AA, Albooai OK, Yasein N, El-Oudah JM (2009) Prevalence and factors associated with overweight and obesity among Jordan university students. J Bio Sc 9: 738-45.

52. Chen JJ, Pegram LI, Adcock KR, Johnson MR (2014) Prevalence of Obesity and Chronic Disease Risks among Ethnic Groups of College Students in Southeast Texas. Austin J Nutr Metab 1: 6.

53. Ranasinghe P, Cooray DN, Jayawardena R, Katulanda P (2015) The influence of family history of hypertension on disease prevalence and associated metabolic risk factors among Sri Lankan adults. BMC Public Health 15: 576.

54. Yoon PW, Scheuner MT, Peterson-Oehlke KL, Gwinn M, Faucett A, et al. (2002) Can family history be used as a tool for public health and preventive medicine? Genet Med 4: 304-10.

55. Ko KP, Oh DK, Min H, Kim CS, Park JK, et al. (2012) Prospective study of optimal obesity index cutoffs for predicting development of multiple metabolic risk factors: the Korean genome and epidemiology study. J Epidemiol 22: 433-9.

56. Tan CE, Ma S, Wai D, Chew SK, Tai ES (2004) Can we apply the National Cholesterol Education Program Adult Treatment Panel definition of the metabolic syndrome to Asians? Diabetes Care 27: 1182-6.

57. Examination Committee of Criteria for 'Obesity Disease' in Japan; Japan Society for the Study of Obesity (2002) New criteria for 'obesity disease' in Japan. Circ J 66: 987-92.

58. Jakicic JM, Otto AD (2005) Physical activity considerations for the treatment and prevention of obesity. Am J Clin Nutr 82: 226S-9S.

59. Anokye N, Trueman P, Green C, Pavey TG, Taylor RS (2012) PA and Health Related Quality of Life. BMC Public Health 12: 624.

60. Ladabaum U, Mannalithara A, Myer PA, Singh G (2014) Obesity, abdominal obesity, PA, and caloric intake in US adults: 1988 to 2010. Am J Med 127: 717-27.

61. Schmitz KH, Jacobs DR Jr, Leon AS, Schreiner PJ, Schreiner PJ, Sternfeld B (2000) Physical activity and body weight: associations over ten years in the CARDIA study. Coronary Artery Risk Development in Young Adults. Int J Obes Relat Metab Disord 24: 1475-87.

62. Amalba A, Oladele J, Mwinlenaa PP, Mogre V (2012) Impact of PA levels and diet on central obesity among civil servants in Tamale metropolis. J. Med. Biomedical Sci 1: 1-9.

63. Petersen L, Schnohr P, Sorensen T (2004) Longitudinal study of the long-term relation between PA and obesity in adults. Int J Obes Relat Metab Disord 28: $105-12$.

64. Lyznicki JM, Young DC, Riggs JA, Davis RM (2001) Obesity: assessment and management in primary care. Am Fam Physician 63: 2185-96.

65. Wardle J, Guthrie C, Sanderson S, Birch LL, Plomin R (2001) Food and activity preferences in children of lean and obese parents. Int J Obes Relat Metab Disord 25: $971-7$.

66. Davison KK \&Birch LL (2002) Obesogenic families: parents' PA and dietary intake patterns predict girls' risk of overweight. Int J Obes Relat Metab Disord 26: 1186-93.

67. Samir N, Mahmud S, Khuwaja AK (2011) Prevalence of physical inactivity and barriers to PA among obese attendants at a community health-care center in Karachi, Pakistan. BMC Res Notes 4: 174.

68. Slentz CA, Duscha BD, Johnson JL, Ketchum K, Aiken LB, et al. (2004) Effects of the amount of exercise on body weight, body composition, and measures of central obesity: STRRIDE--a randomized controlled study. Arch Intern Med 164: 31-9.

69. Ross R, Janiszewski PM (2008) Is weight loss the optimal target for obesity-related cardiovascular disease risk reduction? Can J Cardiol 24: 25D-31D.

70. Ross R, Janssen I, Dawson J, Kungl AM, Kuk JL, et al. (2004) Exercise-induced reduction in obesity and insulin resistance in women: a randomized controlled trial. Obes Res 12: 789-98. 


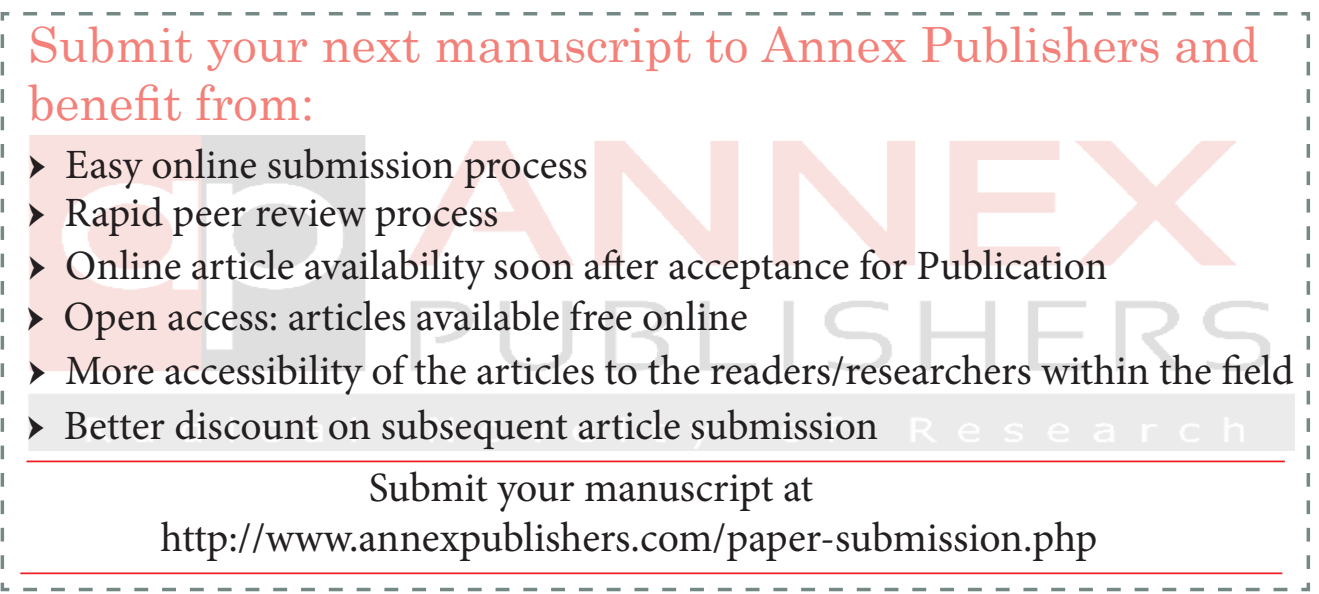

